\title{
LA ENSEÑANZA DE LAS CIENCIAS
}

Entre el 25 de noviembre y el 19 de diciembre de 1980 se desarrolló en las ciudades de Bogotá, Sincelejo y Cali, un ciclo de cuatro seminarios sobre la enseñanza de las ciencias. En su organización participaron el Instituto Italiano de Cultura, la División de Ciencias de la Universidad de Sucre, el Multitaller de Materiales Didácticos de la Universidad del Valle y, en la promoción, realización y coordinación general, el Centro de Investigaciones de la Universidad Pedagógica (CIUP) y el Fondo Colombiano de Investigaciones Científicas y Proyectos Especiales Francisco José de Caldas (COLCIENCIAS) como actividad de su programa conjunto sobre Investigaciones e Innovaciones en la Enseñanza de las Ciencias.

La financiación del evento se hizo posible gracias a la colaboración tanto de las entidades ya mencionadas como del Ministerio de Instrucción Pública de Italia, el Instituto Colombiano para el Fomento de la Educación Superior (ICFES) y el Programa Col 76/005 para el mejoramiento de la investigación y la enseñanza de las ciencias básicas.

Las universidades italianas de Roma y Pavía contribuyeron de manera igualmente significativa, facilitando la participación de once profesores pertenecientes a los grupos de investigación "Universidad Escuela" y "Laboratorio Didáctico" en el caso de la primera y al "Centro Didáctico" en el de la segunda.

Los cuatro seminarios contaron con la participación de más de 250 personas. Entre ellas: investigadores en el área; docentes de nivel universitario o medio interesados en el tema, ya por las exigencias de su práctica académica, ya por su vinculación a proyectos innovativos; funcionarios públicos cuya actividad se relaciona directamente con programas de desarrollo curricular o de capacitación de docentes en entidades gubernamentales tales como Ministerio de Educación, ICFES y Secretarías de Educación y funcionarios de empresas editoriales.

El trabajo de cada uno de los seminarios efectuados giró en torno a conferencias y grupos de trabajo con sus respectivos debates, excepción hecha del de Sincelejo, en cuyo desarrollo y debido a las características particulares de los asistentes - docentes en ciencias a nivel secundario, en su mayoría- se adoptó, principalmente, la modalidad de discusiones en pequeños grupos y enmarcados en el diseño general de un curso de actualización.

Tres objetivos básicos se perseguían con la realización de estos seminarios:

1. Continuar, estimular y ampliar la discusión que sobre la enseñanza de las ciencias se ha generado en el país. En efecto, desde algunos años el tema viene captando la atención de sociedades científicas, de docentes de los niveles medio y universitario y de algunas oficinas gubernamentales, originando un debate cuyos primeros resultados ya se observan en la conformación de diversos grupos de investigación, dedicados al estudio de algunos de los problemas señalados como los más relevantes en el campo y a sus posibles soluciones.

No obstante, hasta el momento la reflexión se ha centrado en aspectos tales como de política educativa y metodología de la enseñanza de cada una de las disciplinas científicas, descuidando aspectos de tanta o mayor importancia como serían los 
referentes a la didáctica de las diferentes disciplinas, o la relación historia-didáctica de las ciencias, a la relación desarrollo cognoscitivo-conocimiento científico y algunos otros de carácter aún más especializado.

Los seminarios pretendían entonces, inducir una apertura de la investigación realizada en Colombia hacia esos nuevos tópicos señalados $\mathrm{y}$, en general, abrir nuevas perspectivas temáticas y metodológicas a los interesados en la investigación sobre la enseñanza de la ciencia. Este propósito obedeció a la consideración de que el espectro de problemas por ser abordado es enormemente superior en cantidad, cobertura y profundidad al asumido hasta ahora por los estudios adelantados. Dicho espectro debería ir desde la investigación sobre el contexto social en el que se desenvuelve la enseñanza de las ciencias (su papel actual, su futuro, sus prioridades, etc.), pasar por la investigación pedagógica y didáctica y llegar hasta la investigación para la generación de modelos sobre temáticas específicas (en condiciones igualmente específicas) de carácter innovador.

2. La estrechez del debate no se circunscribe, sin embargo, a lo limitado de sus temas. Otra de sus grandes limitaciones y que necesariamente deberá obviar-se, la constituye el hecho de que no se han hecho partícipes en el mismo todas las partes que deberían y podrían estar interesadas: Estado, investigadores, autores de textos, editoriales, docentes, padres y alumnos. La heterogénea composición de la participación en los seminarios respondió precisamente al objetivo de tratar de aprovechar la oportunidad para establecer, por lo menos, algunos mecanismos iniciales de comunicación e integración entre los diversos estamentos que de alguna manera tienen injerencia en el proceso de enseñanza de las ciencias en el país.

3. Por último, dada la escasa tradición investigativa del medio colombiano, la limitación de sus recursos financieros y aun lo reducido de la infraestructura humana con la cual cuenta para su realización, una de las necesidades más apremiantes de la investigación en todo campo y en el de la enseñanza de las ciencias en particular, es la de hacer un aprovechamiento óptimo de los recursos evitando duplicidades inútiles, agilizando el flujo de información entre investigadores y entre éstos y las oficinas gubernamentales con injerencia directa en el aparato escolar. De allí que la intención de romper el aislamiento en el que se encuentra el investigador colombiano frente a sus colegas tanto nacionales como extranjeros, se definiese como otro de los objetivos prioritarios de los seminarios.

Las conferencias dictadas durante los cuatro seminarios así como los principales aspectos de las discusiones plenarias y de los grupos de trabajo aparecerán en las memorias cuya edición se adelanta en la actualidad.

De una manera muy esquemática y para información inicial de los lectores, las conferencias y discusiones giraron principalmente sobre los tópicos siguientes:

1. Conocimiento común y conocimiento científico en adultos y niños.

Existe una brecha, cada vez mayor, entre el desarrollo científico y tecnológico de las sociedades modernas y el nivel de comprensión de la ciencia y la tecnología por parte de la gran masa de nuestra población. 
¿Cuál es ese conocimiento científico necesario para vivir en una sociedad moderna y que debe convertirse en conocimiento de sentido común de nuestra población? ¿Cuál es el conocimiento común actual de las ciencias en nuestra población adulta, en nuestros maestros, en nuestros niños?

\section{Educación y Ciencia.}

Dos puntos del debate: a) ¿La ciencia debe ser entendida como un "estado" de conocimientos y la Educación como un "proceso" hacia el conocimiento? b) ¿La actividad escolar referida a las ciencias solo puede adquirir sentido para el alumno en la medida en que esta actividad incluya como elemento fundamental la relación (afectiva, cognitiva, socio-cultural) con el mundo a través de las ciencias? ¿La lógica de la eficiencia imperante en nuestra educación ha impedido la formación de una actitud científica capaz de asumir los saberes y las actividades en función de la transformación del mundo?

\section{Desarrollo Cognoscitivo y conocimiento científico.}

Una idea de base: la investigación educativa y la didáctica deben tender a identificar o por lo menos a recoger los modos de pensar de los alumnos, y no sólo eso, sino sus modos de pensar en relación a contenidos específicos, a contextos disciplinarios especiales.

\section{Historia, Epistemología y Didáctica de las Ciencias.}

¿Qué relación existe entre ciencia, filosofía de la ciencia y didáctica?

¿Qué contribuciones pueden hacer la historia y la epistemología de las ciencias a una enseñanza innovativa de ellas?

¿Se hace necesario el "laboratorio" histórico en la enseñanza de las ciencias?

5. Situación de la enseñanza de las ciencias tanto a nivel primario como secundario y universitario.

6. Problemas y alternativas en la construcción de currículos para la enseñanza de las ciencias.

7. Problemas y alternativas en la capacitación y actualización de docentes para la enseñanza de las cien"ias.

8. La cooperación a nivel nacional e internacional en la investigación sobre la enseñanza de las ciencias.

Mirados en su conjunto, los cuatro seminarios ofrecen un balance satisfactorio. La acogida y el interés que despertaron y mantuvieron entre los asistentes puede ser un indicador de que con ellos se contribuyó a satisfacer algunas de las inquietudes y necesidades expuestas en otras reuniones y discusiones.

La confrontación de nuestras experiencias investigativas con la de los profesores italianos permitió valorar nuestros logros e identificar deficiencias y vacíos. Una vez más 
se puso de presente la importancia y la necesidad de eventos de esta naturaleza: de intercambio de experiencias, de colaboración, de apertura al mundo.

Uno de los mayores logros de los seminarios fue el haber ganado conciencia sobre la importancia que tiene la investigación en la didáctica de las ciencias como base de cualquier esfuerzo para el mejoramiento de su enseñanza.

Nuestra enseñanza actual de las ciencias, inclinada más hacia la transmisión de resultados científicos que hacia la formación de una verdadera actitud científica, obliga a la comunidad investigativa a dedicar su esfuerzo reflexivo sobre una educación en ciencias más abierta, más creativa, más ligada a los problemas y realidades de nuestra sociedad, más dispuesta a la innovación y al cambio como lo exige el acelerado desarrollo de la ciencia y la tecnología.

Esta situación y este posible y factible dominio de trabajo investigativo pusieron de presente una de las prioridades más grandes del país: La investigación orientada a sentar las bases y a generar modelos para una formación y capacitación de docentes capaces de hacer realidad esa nueva educación en ciencias.

Igualmente merece destacarse en esta síntesis inicial y general de resultados, el reconocimiento que los participantes hicieron de la necesidad de incorporar a docentes de educación media y primaria en todos los proyectos de investigación. Sin una más estrecha relación entre la Universidad y la escuela, entre la "teoría"de la primera y la "práctica" de la segunda, pierde la investigación educativa su sentido de transformación.

\section{COMENTARIO GENERAL DE LA DOCTORA MATILDE VICENTINI MISSONI ${ }^{206}$}

Una mirada hacia atrás, a las cuatro semanas de trabajo en Colombia, nos permite clarificar algunos aspectos de esta clase de intercambio bilateral de ideas y experiencias. Esto puede ser útil comunicarlo a otros interesados en los problemas de la educación en el mundo.

El largo período de permanencia en diferentes lugares de un país extranjero, da una buena oportunidad para tener una visión más profunda de los antecedentes socioculturales propios de los problemas educativos colombianos. En efecto, mientras las dos primeras semanas en Bogotá nos mostraron los aspectos más generales y amplios de la problemática, la tercera semana en Sincelejo nos permitió una confrontación concreta con los problemas más palpitantes de la preparación, formación y capacitación de maestros en servicio, la cual sentó las bases para un entendimiento más profundo, durante la última semana en Cali, de las experiencias de los investigadores colombianos.

Para los once investigadores italianos, identificados por un interés común en la educación pero manteniendo sus intereses específicos así como sus puntos de vista personales, la participación en los seminarios propició discusiones entre nosotros sobre

\footnotetext{
${ }^{206}$ La Doctora Vicentini formó parte del grupo de profesores italianos participantes en los seminarios. Trabaja en el Instituto de Física de la Universidad de Roma. Pertenece al grupo de investigación "Laboratorio Didáctico" y dirige el grupo "UniversidadEscuela" de la misma universidad.
} 
varios de los tópicos tratados y permitió una reflexión sobre nuestra propia experiencia investigativa y sobre los problemas educativos de nuestro propio país.

Se captaron similitudes y diferencias. Un ejemplo de estas últimas es la política educativa de los gobiernos de Italia y de Colombia. Un ejemplo de similitudes: el informe italiano sobre el conocimiento de sentido común en los adultos (Vicentini-Noce) y el in forme colombiano sobre la relación entre pre-teorías y teorías científicas (segura). Estos dos ejemplos ponen de presente la importancia de la investigación educativa y de su divulgación para el diseño de proyectos curriculares, los cuales deben tener en cuenta para su aplicación en una realidad social dada, los lineamientos impuestos por las políticas de los gobiernos.

Álvaro Pantoja Velásquez 